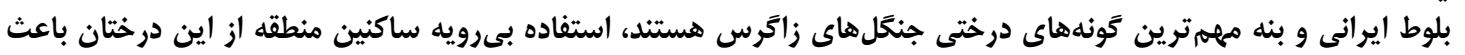

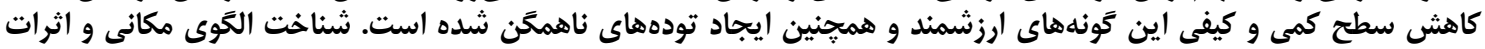

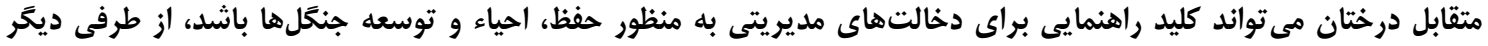

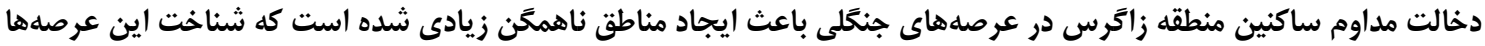

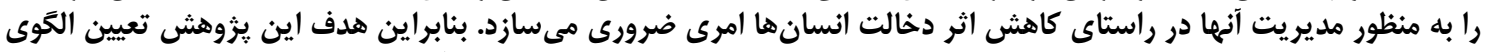

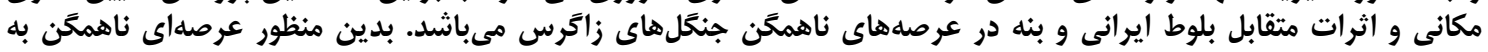

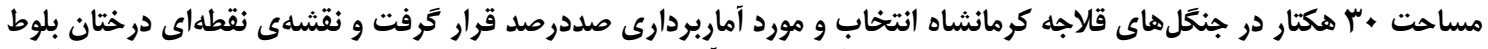

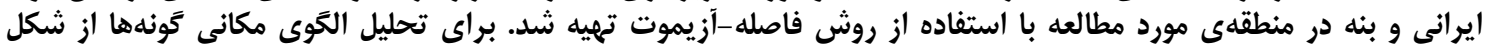

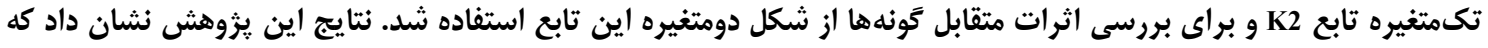

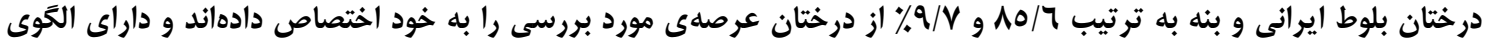

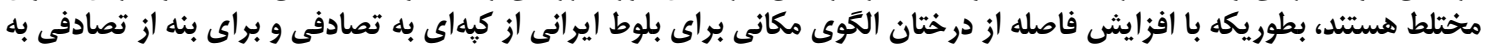

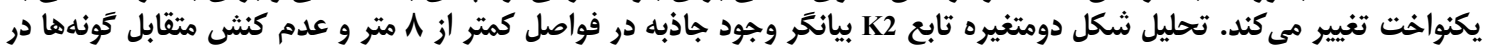
ساير فواصل است.

وازههاى كليدى: نقشه نقطهاى، توده ناهمًَن، روش فاصله-آزيموت، حدود مونت كارلو، التوى كِيداى

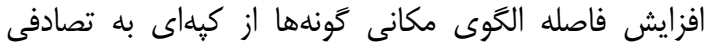

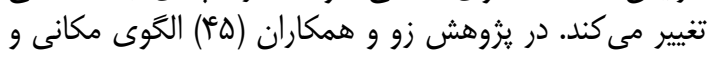

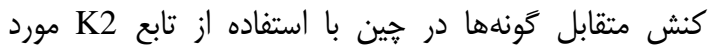

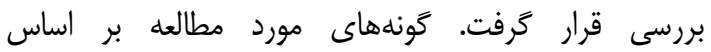

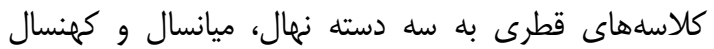

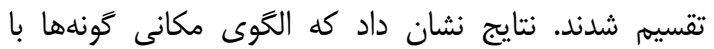

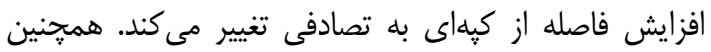

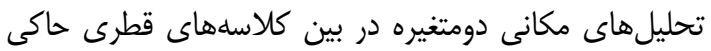

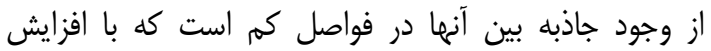

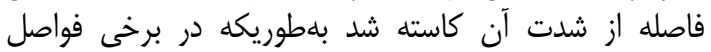

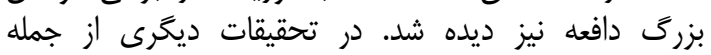

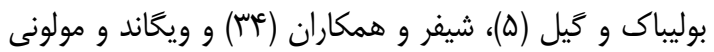

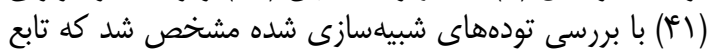

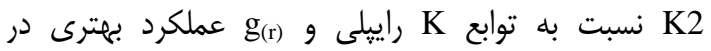

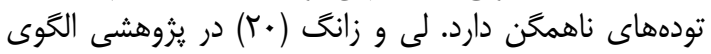

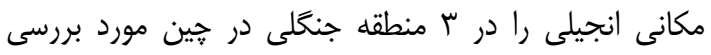

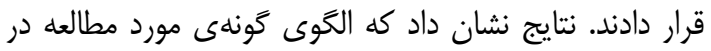

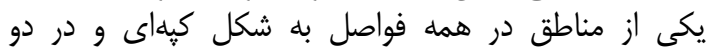

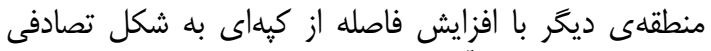

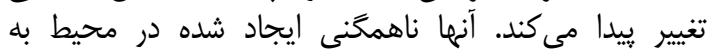

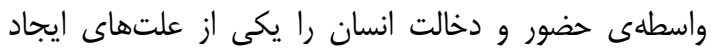

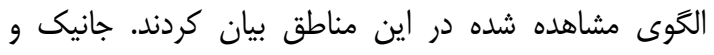

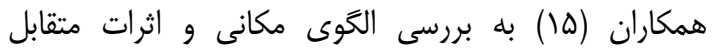

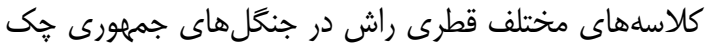

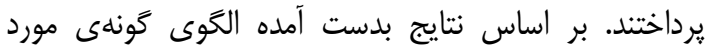

مقدمه

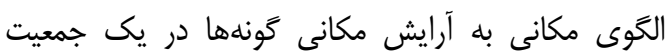

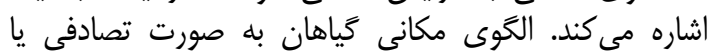

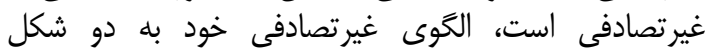

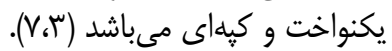

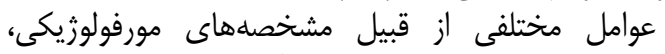

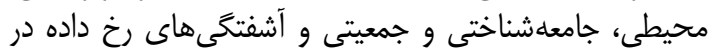

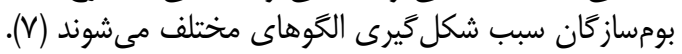

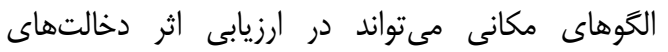

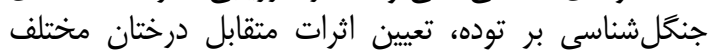

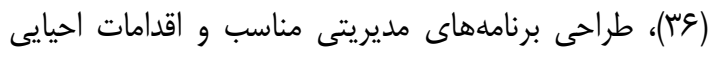

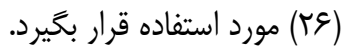

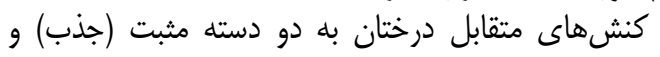

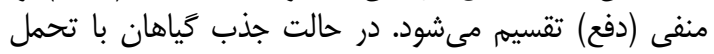

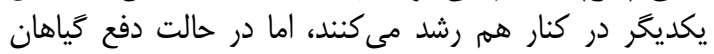

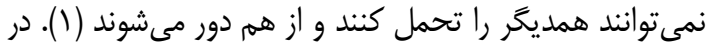

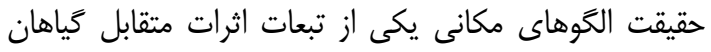

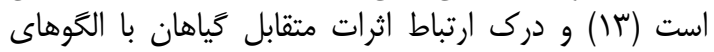

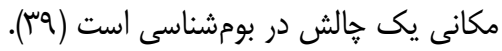

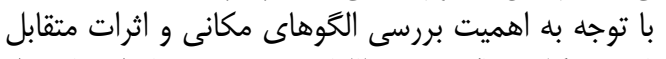

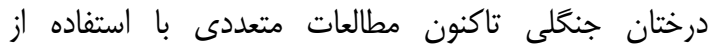

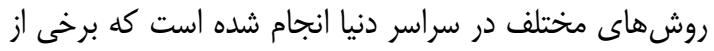

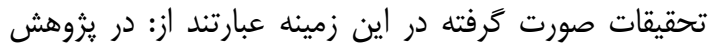

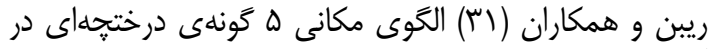
آمريكا با استفاده از تابع K2 بررسى شد. نتايج نشان داد كه دان با دان 
صحيح و بهرهبردارى بهينه از اين جنكلها بلمناريظور توليد

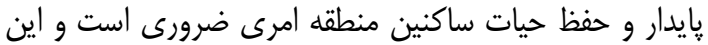

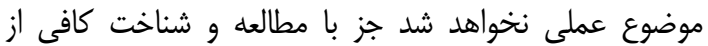

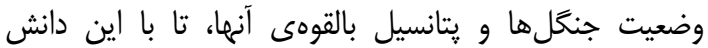

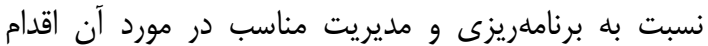

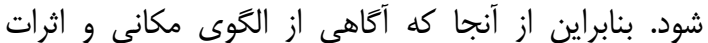

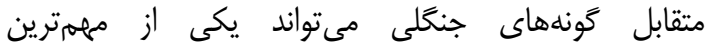

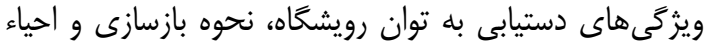

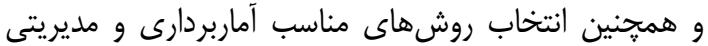

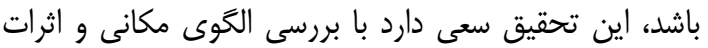

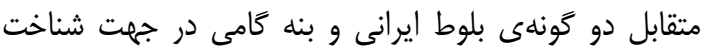

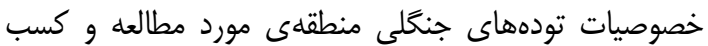
اطلاعات مورد نياز به منظور مديريت آنها بردارد.

\section{مواد و روشها موردال منطقة مورد مطالعه}

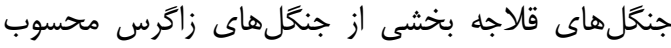

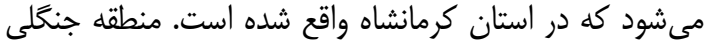

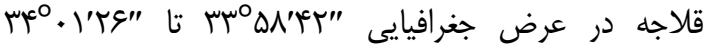

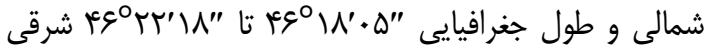

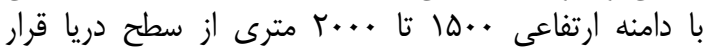

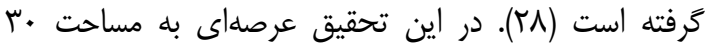

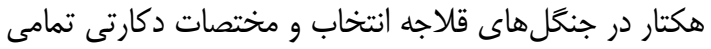

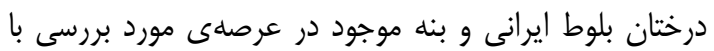

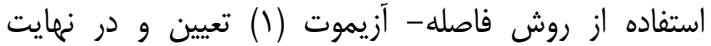

نقشهى نقطهاى درختان در منطقهى مورد مطالعه تهييه شد.

تحليل دادهها

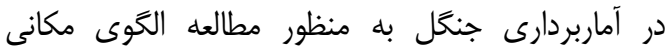

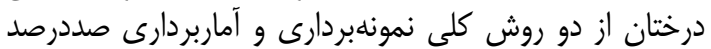

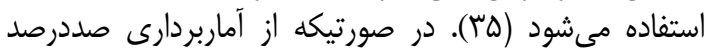

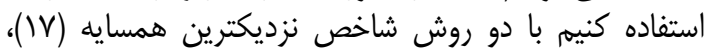

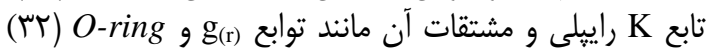

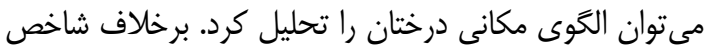

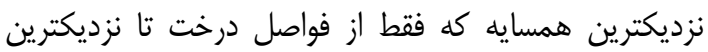

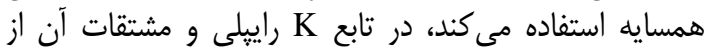

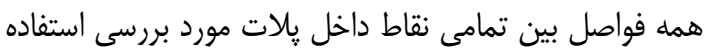

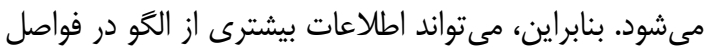

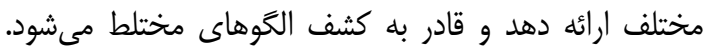

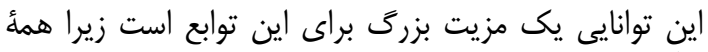

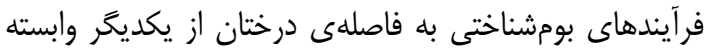

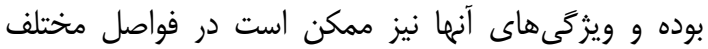

تغيير كند (•)

تابع K ايديلى

ايدة اصلى تابع K راييلى تعداد نقاط موجود در داخل دايره با

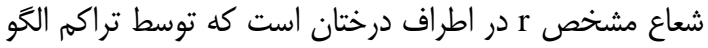

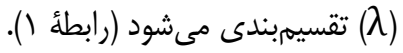

$$
\hat{K}_{(r)}=A \sum_{i}^{n} \sum_{\neq j}^{n} w_{i j} I_{r}(i, j) / n^{2}
$$

مطالعه در هملى كلاسهاى قطرى تا فاصلهى هـ مترى از

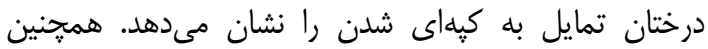

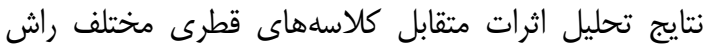

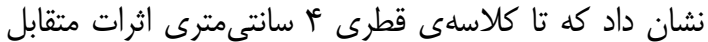

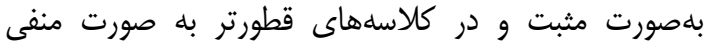

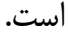

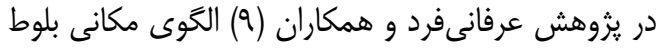

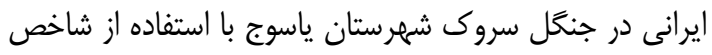

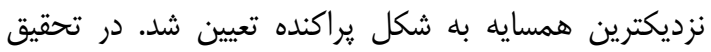

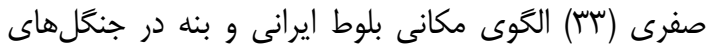

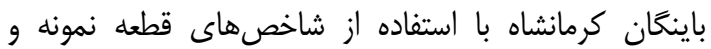

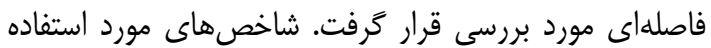

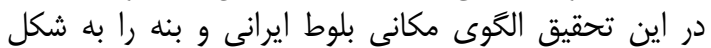

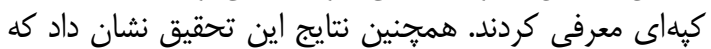

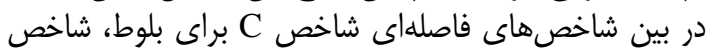

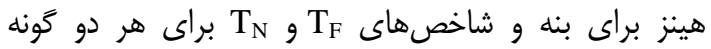

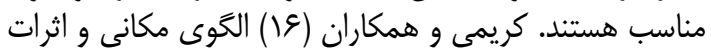

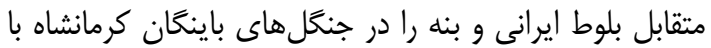

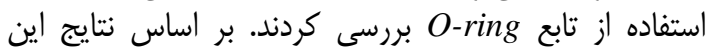

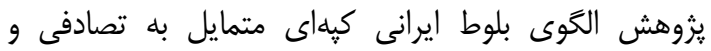

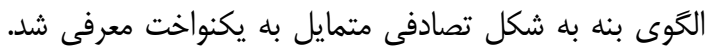

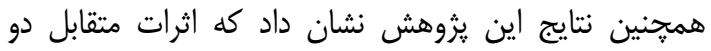

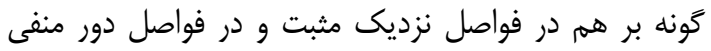

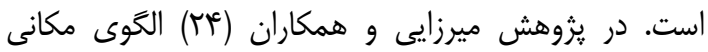

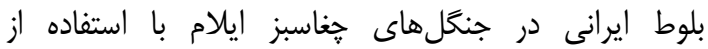

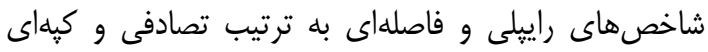

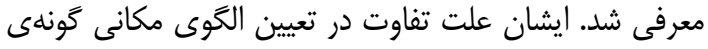

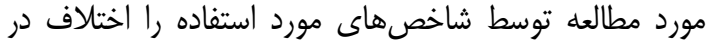

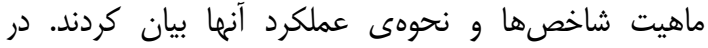

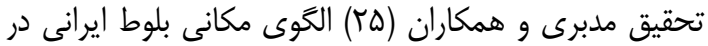

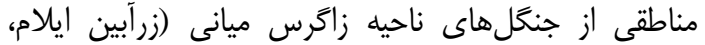

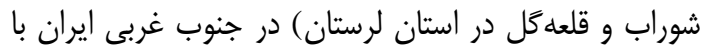

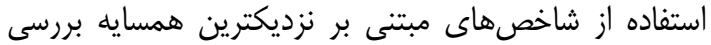

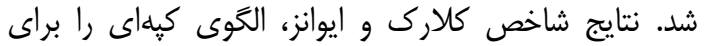
مناطق شوراب و قلعهكل و الكوى يكنواخت رات را براى منطقه

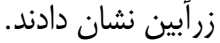

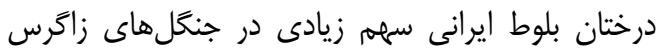

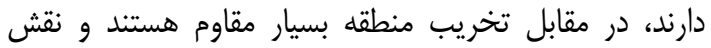

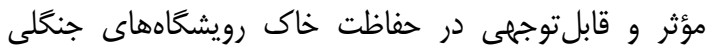

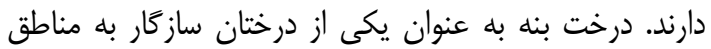

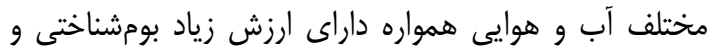

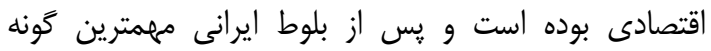

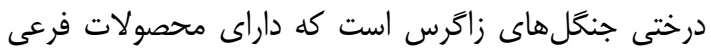

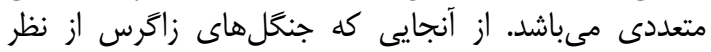

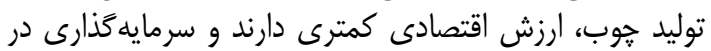

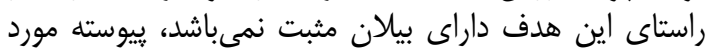

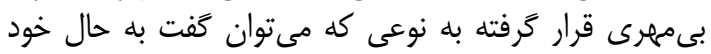

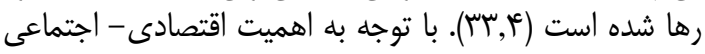

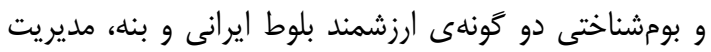




$$
K 2(r)=\frac{\hat{g}(r+\Delta r)-\hat{g}(r-\Delta r)}{2 \Delta r}
$$

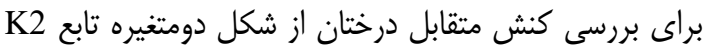

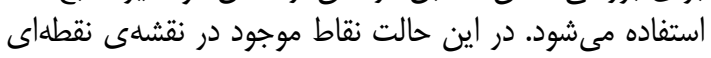

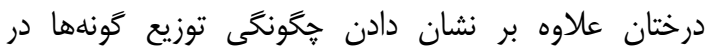

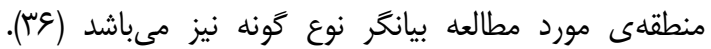

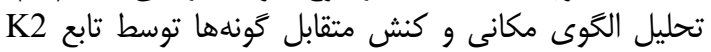

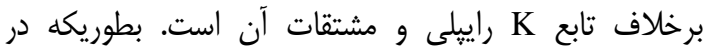

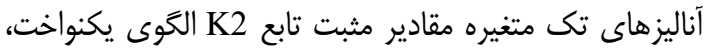

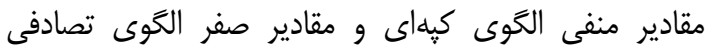

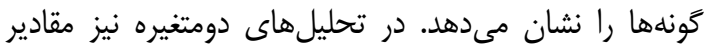

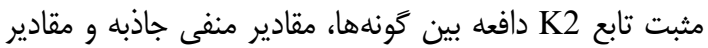
صفر استقلال كونهها را بيان مى كند (جدول مقادي مان ).

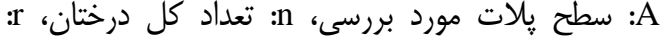

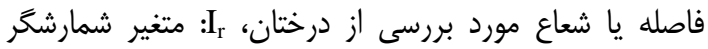

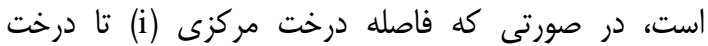

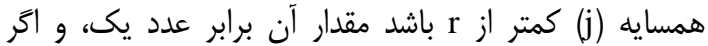

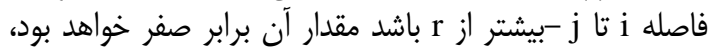

و

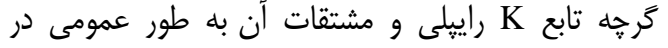

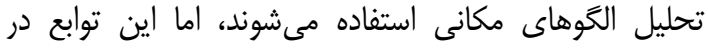

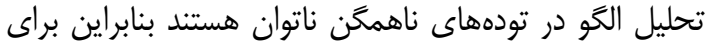

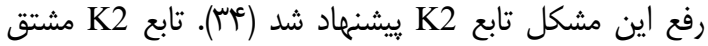
دوم تابع K راييلى است (مشتق اول تابع (r)

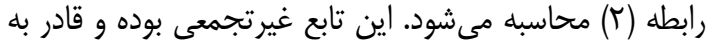

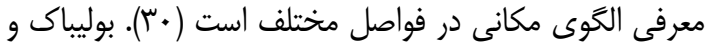
و كيل (ه) نيز كارايى مثبت تابع K2 را تأييد كردند.

جدول ا- تعيين الخوى مكانى و كنش متقابل درختان با استفاده از تابع K2 Table 1. Determination of spatial pattern and interaction of trees using the K2 function

\begin{tabular}{|c|c|c|}
\hline كنش متقابل & الكوى مكانى & K2 \\
\hline جاذبه بين دو كَروه & كيهاى & $\mathrm{K} 2<$ • \\
\hline استقلال بين دو گروه & تصادفى & $\mathrm{K} 2=\cdot$ \\
\hline دافعه بين دو كروه & يكنواخت & $\mathrm{K} 2>$. \\
\hline
\end{tabular}

Programita (OThorsten Wiegand 2014) نرمافزار

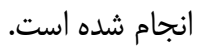

نتايج و بحث

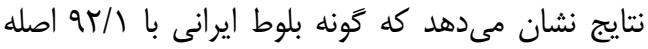

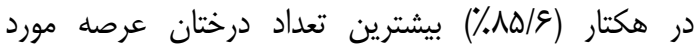

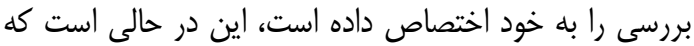

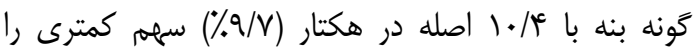

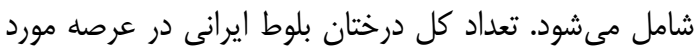

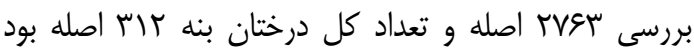

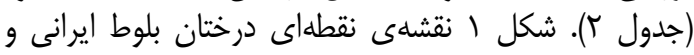

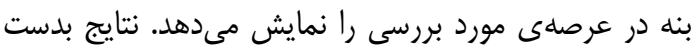

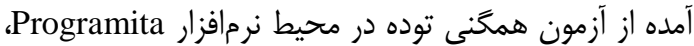
بيانكر ناهمخنى تودهى مورد مطالعه است (شكل ك ؟).

Table 2. Frequency and density of species in the study area

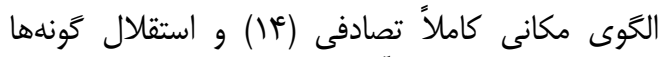

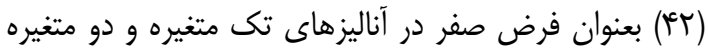

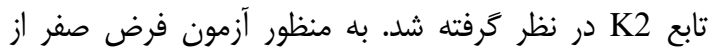

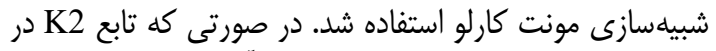

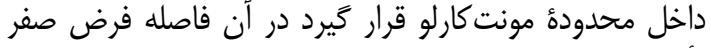

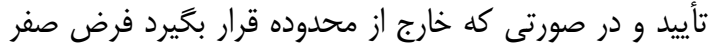

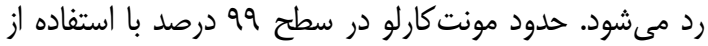

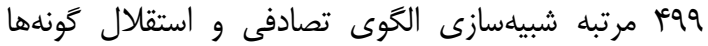

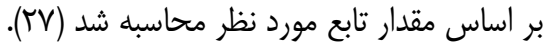

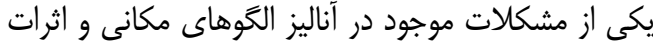

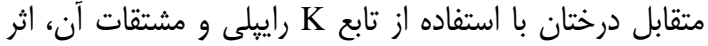

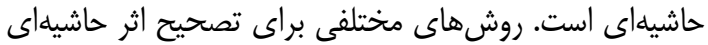

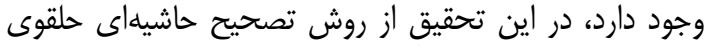

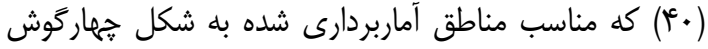

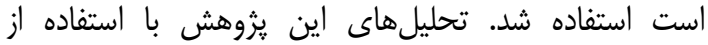

جدول r- فراوانى و تعداد در هكتار كونهها در منطقهى مورد مطالعه

\begin{tabular}{|c|c|c|c|}
\hline تعداد در هكتار & درصد فراوانى & فراوانى مطلق & كونه \\
\hline $9 / / 1$ & $\Lambda \Delta / \bar{G}$ & TVGT & بلوط ايرانى \\
\hline $1 . / 4$ & $9 / v$ & זוץ & بنه \\
\hline$\Delta / 1$ & $r / V$ & IDF & ساير \\
\hline
\end{tabular}




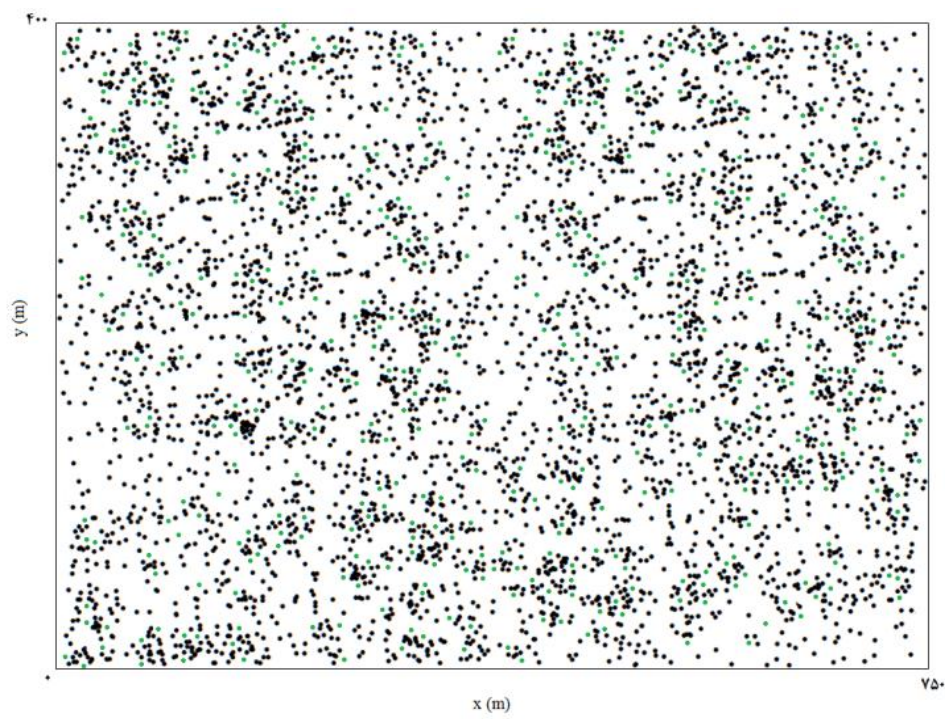

$x(m)$

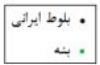

شكل (- نقشهى نقطهاى درختان بلوط ايرانى و بنه در منطقهى مورد مطالعه

Figure 1. Point map of $Q$. brantii and P. atlantica trees in the study area

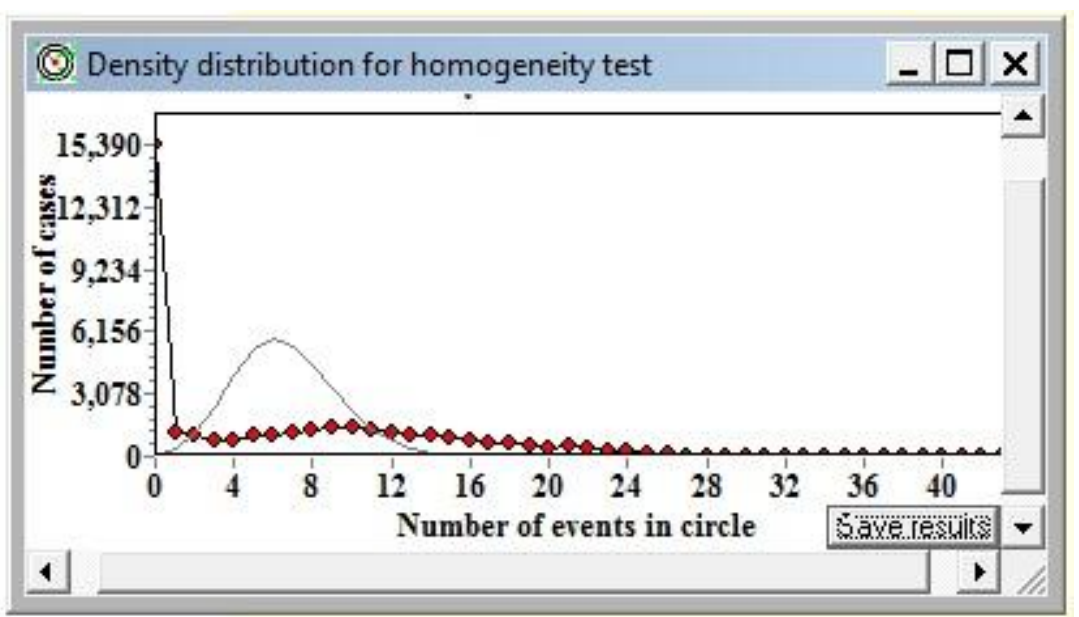

شكل r- آزمون همخنى براى درختان بلوط ايرانى و بنه

Figure 2. Homogeneity test for $Q$. brantii and $P$. atlantica trees

در اكثر فواصل تابع K2 در داخل محدودهى مونت كارلو قرار

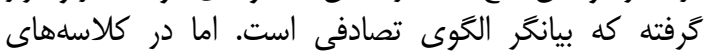

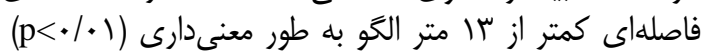

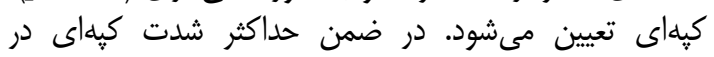

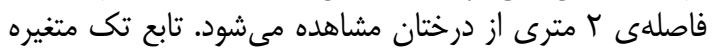

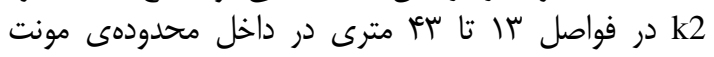

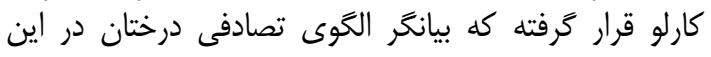

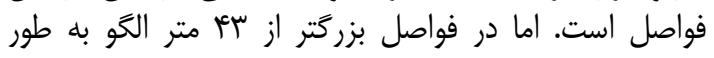

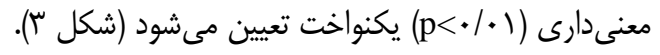

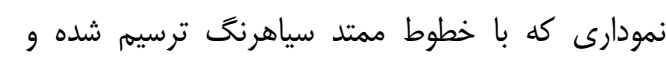

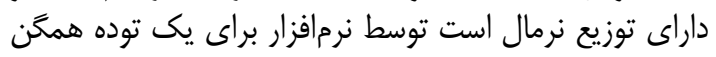

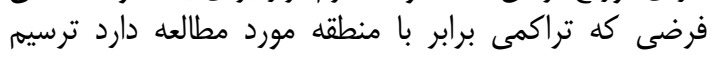

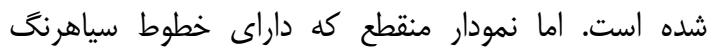

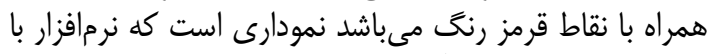

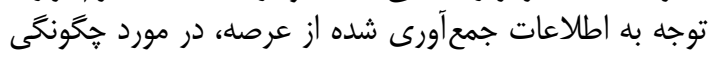

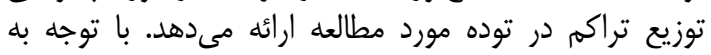

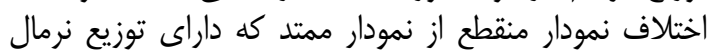

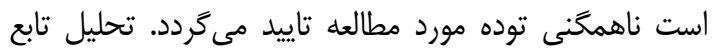
تك متغيره K2 براى درختان بلوط ايرانى و بنه نشان داد كه تحان 


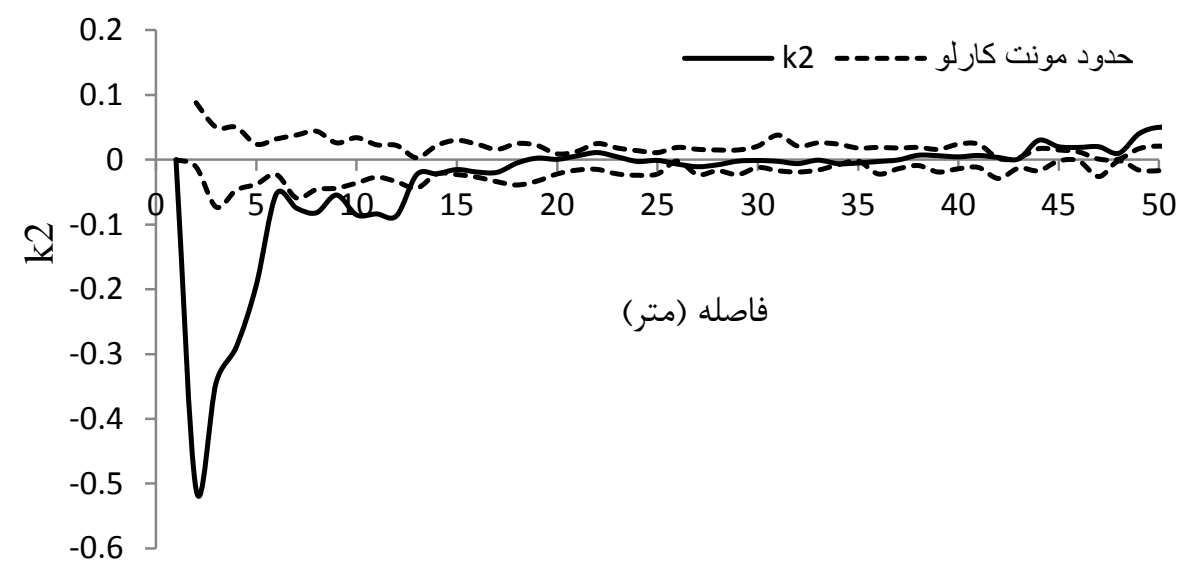

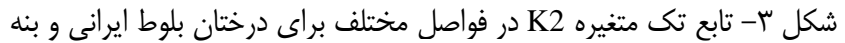

Figure 3. K2 univariate function at different distances for $Q$. brantii and P. atlantica trees

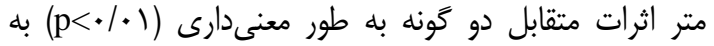

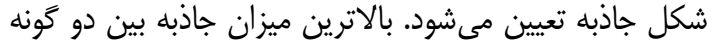

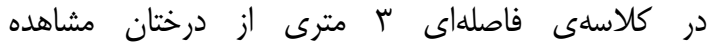

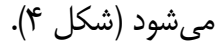

تحليل تابع دومتغيره K2 نشان داد كه در اكثر فواصل

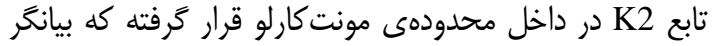

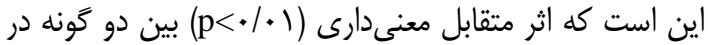

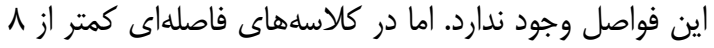

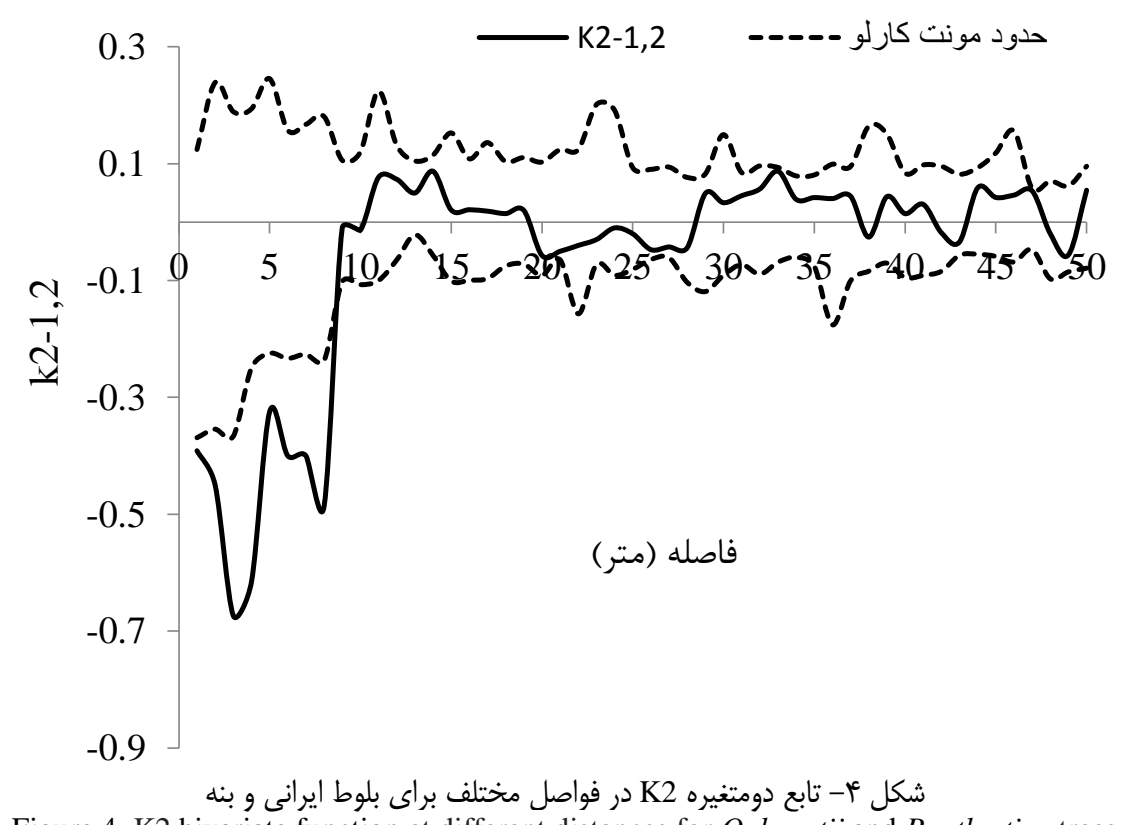

Figure 4. K2 bivariate function at different distances for $Q$. brantii and $P$. atlantica trees

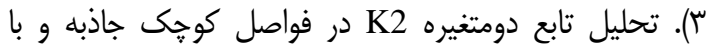

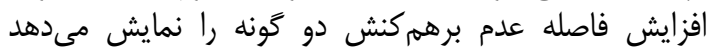

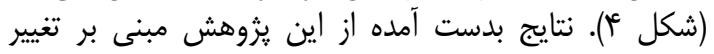

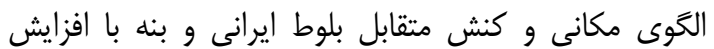

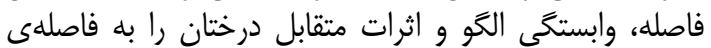

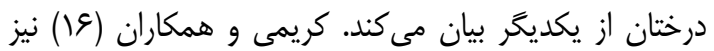
در يثوهش خود اين وابستخى را تأييد كردند.

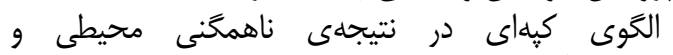

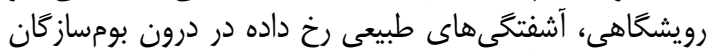

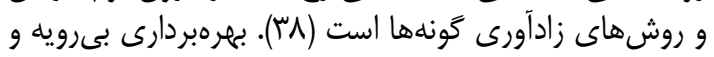

دستيابى به اطالاعات موجود در الكوى مكانى درختان

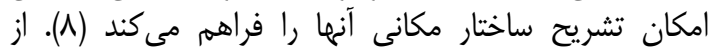

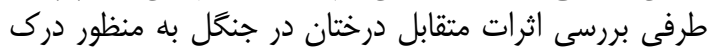

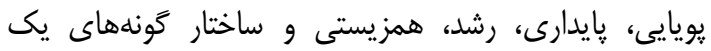

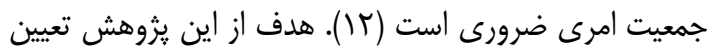

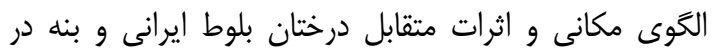

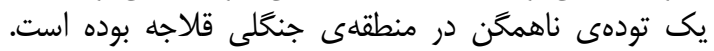

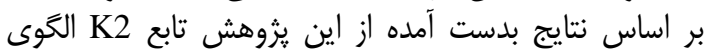

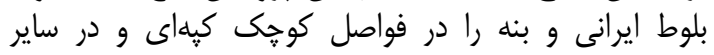
فاصلهها به صورت تصادفى و يكنواخت معرفى مى كند (شكل 
كرفتن تعداد بيشتر درختان در واحد سطح در فواصل كوجى

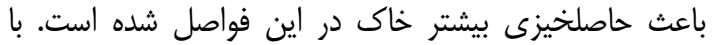

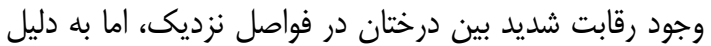

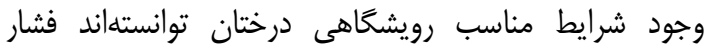

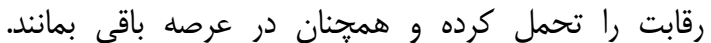

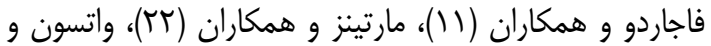

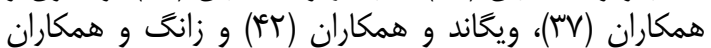

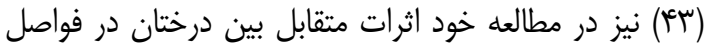

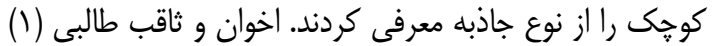

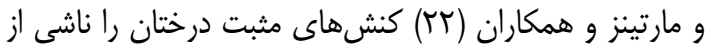

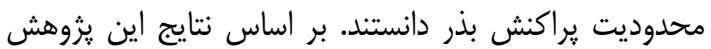

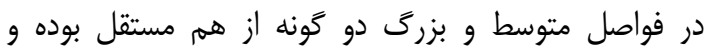

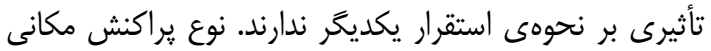

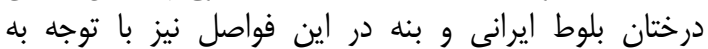

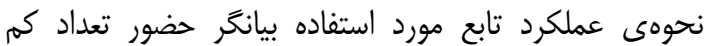

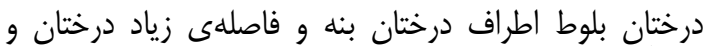

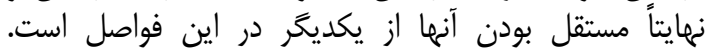

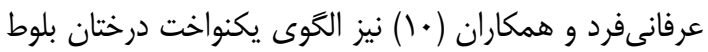

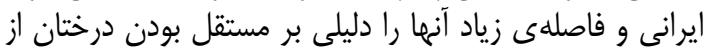

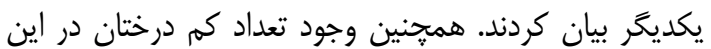

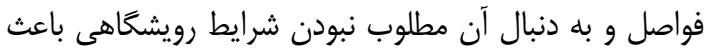

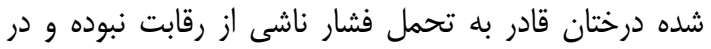

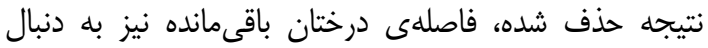

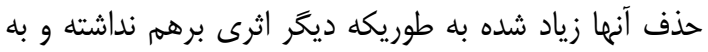

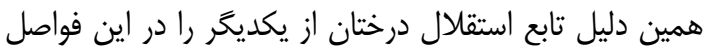

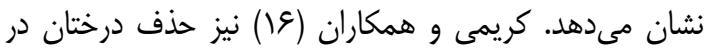

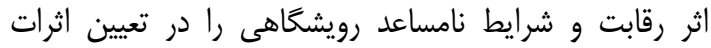

متقابل درختان در فواصل متوسط و بزر باعلى موثر دانستند.

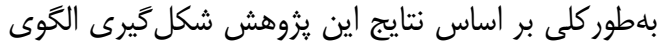

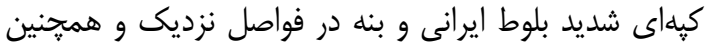

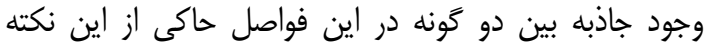

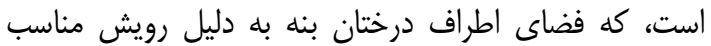

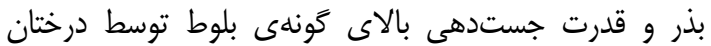

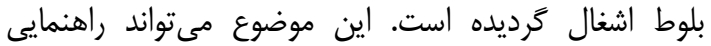

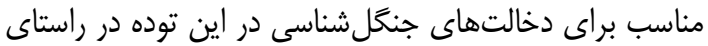

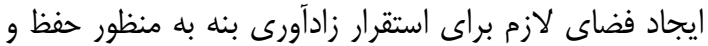
احياء اين كونه ارزشمند باشد.

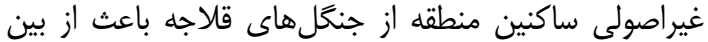

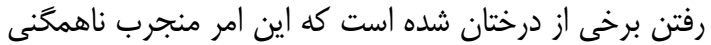

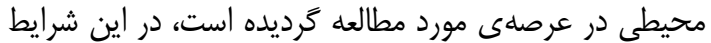

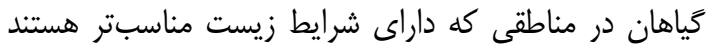

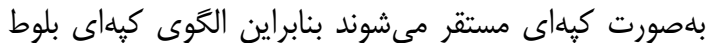

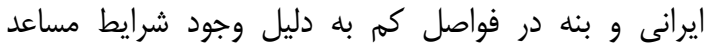

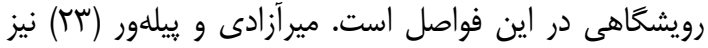

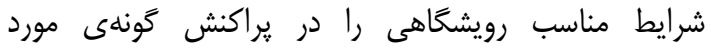

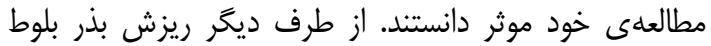

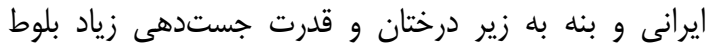

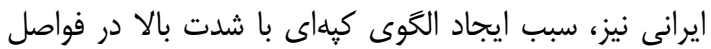

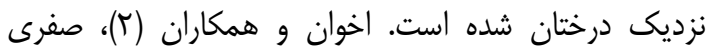

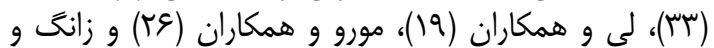

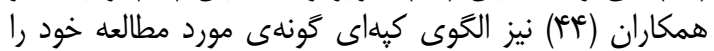
به دليل نحومى زادآورى آنها معرفى كردندي

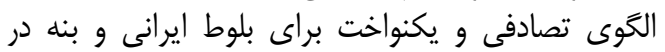

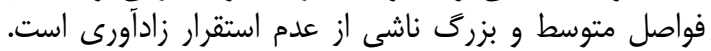

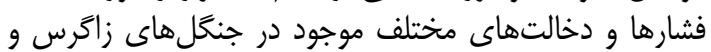

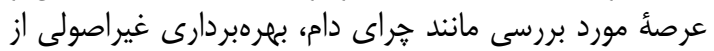

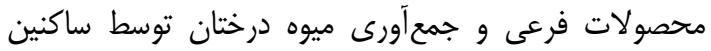

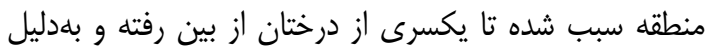

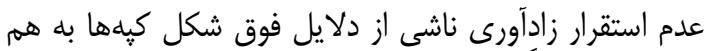

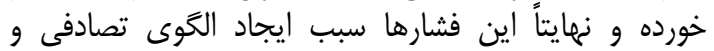

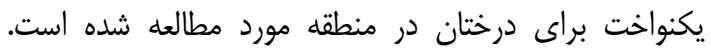

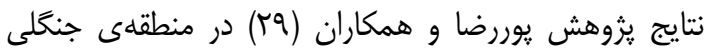

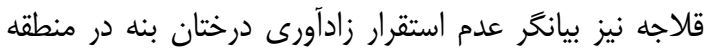

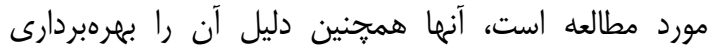

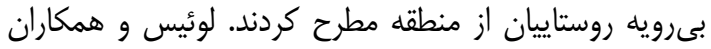

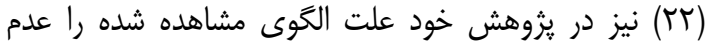

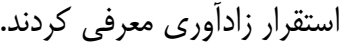

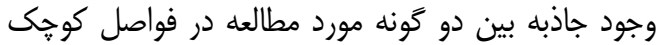

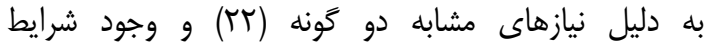

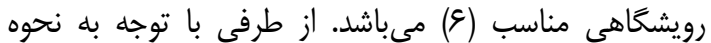

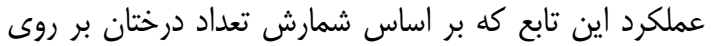

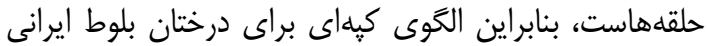

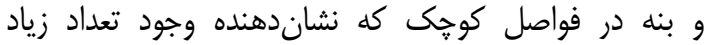

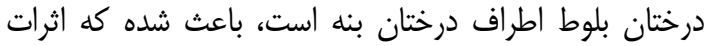
متقابل درختان در اين فواصل به شكل جاذ باذبه بيان شود. قرار 
منابع

. Akhavan, R. and Kh. Sagheb-Talebi. 2012. Application of bivariate Ripley's K- function for studying competition and spatial association of trees (Case study: intact Oriental beech stands in Kelardasht). Iranian Journal of Forest and Poplar Research, 19(4): 632-644 (In Persian).

2. Akhavan, R., Kh. Sagheb-Talebi, M. Hassani and P. Parhizkar. 2010. Spatial patterns in untouched beech (Fagus orientalis Lipsky) stands over forest development stages in Kelardasht region of Iran. Iranian Journal of Forest and Poplar Research, 18(2): 322-336 (In Persian).

3. Baddeley, A. 2008. Analysing spatial point patterns in R. Workshop Notes, $171 \mathrm{pp}$.

4. Bagheri, J., A. Salehi and K. Taheri Abkenar. 2014. Effective Factors on Regeneration Establishment and Quantitative and Qualitative Characteristics of Pistacia atlantica in Different Physiographic Conditions (Case Study: Khojir National Park). Iranian Forests Ecology, 2(3): 1-12 (In Persian).

5. Bolibok, L. and W. Gil. 2013. Selected methods for spatial structure analysis of tree stands with heterogeneous tree density. Sylwan, 157(8): 618-629.

6. Chesson, P. 2000. Mechanisms of maintenance of species diversity. Annual Review of Ecology and Systematics, 31: 343-366.

7. Dale, M.R.T. 1999. Spatial Pattern Analysis in Plant Ecology. Cambridge University Press, 327 pp.

8. Erfanifard, Y. and F. Naziri. 2017. Comparison of Ripley's K-, pair correlation, and O-ring functions in spatial pattern analysis of Christ's thorn jujube trees (Ziziphus spina-christi) in Fars province. Forest and Wood Products, 70(1): 1-9 (In Persian).

9. Erfanifard, Y., J. Feghhi, M. Zobeiri and M. Namiranian. 2008. Investigation on the Spatial Pattern of Trees in Zagros Forests. Journal of the Iranian Natural Resource, 60(4): 1319-1343 (In Persian).

10. Erfanifard, Y., L. Zare and J. Feghhi. 2013. Application of Indicators of the nearest neighbor in Iranian oak branches Zagros forests (Quercus brantii var. persica). Journal of Applied Ecology, 2(5): 15-24 (In Persian).

11. Fajardo, A., J. Goodburn and J. Graham. 2006. Spatial patterns of regeneration in managed unevenaged Ponderosa pine/Douglas-fir forests of Western Montana, USA. Forest Ecology and Management, 223: $255-266$.

12. Gray, H. and L. He. 2009. Spatial point pattern analysis for detecting density dependent competition in a boreal chronosequence of Alberta. Forest Ecology and Management, 259: 98-106.

13. Harvey, B.D., A. Leduc, S. Gauthier and Y. Bergeron. 2002. Stand- landscape integration in natural disturbance- based management of the southern boreal forest. Forest Ecology and Management, 155: 369-385.

14. Illian, J., A. Penttinen, H. Stoyan and D. Stoyan. 2008. Statistical Analysis and Modelling of Spatial point patterns. John \& whiley Sons, Ltd, 560 pp.

15. Janík, D., K. Král, D. Adam, L. Hort, P. Samonil, P. Unar, T. Vrska and S. McMahon. 2016. Tree spatial patterns of Fagus sylvatica expansion over 37 years. Forest Ecology and Management, 375: 134-145.

16. Karimi, M., M.R. Pormajidian, H. Jalilvand and A. Safari. 2012. Preliminary study for application of $O$-ring function in determination of small-scale spatial pattern and interaction species (Case study: Bayangan forests, Kermanshah). Iranian Journal of Forest and Poplar Research, 20(4): 608-621 (In Persian).

17. Krebs, C.J. 1999. Ecological Methodology. $2^{\text {nd }}$ edn, Benjamin/Cummings, 620 pp.

18. Letcher, B.H., J.A. Priddly, J.R. Walter and L.B. Crowder. 1998. An individual-based, spatiallyexplicit simulation model of the population dynamic of the endangered red-cockaded wood-pecker, Picoides borealis. Biological Conservation, 86: 1-14.

19. Li, L., Zh. Huang, W. Ye, H. Cao, Sh. Wei, Zh. Wang, L. Lian, I.F. Sun, K. Ma and F. He. 2009. Spatial distribution of $t$ ree species in a subtropical forest of China. Oikos, 118: 495-502.

20. Li, W. and G. Zhang. 2015. Population structure and spatial pattern of the endemic and endangered subtropical tree Parrotia subaequalis (Hamamelidaceae). Flora, 212: 10-18.

21. Luis, M.D., J. Raventos, T. Wiegand and C.H. Hidalgo. 2008. Temporal and spatial differentiation in seedling emergence may promote species coexistence in Mediteraranean fire-prone ecosystems. Ecography, 31: 620-629.

22. Martinez, I., T. Weigand, F. Gonzalez and J.R. Obeso. 2010. Spatial associations among tree species in a temperate forest community in North-western Spain. Forest Ecology and Management, 260: 456465.

23. Mirazadi, Z. and B. Pilehvar. 2013. The Effects of Some Ecological Factors on Myrtus Communis Distribution in Lorestan Province. Iranian Forests Ecology, 1(2): 1-11 (In Persian).

24. Mirzaei, M., A.E. Bonyad and J. Aziz. 2016. Investigation comparison of K-Ripley and distance indices in order to determinate of spatial pattern of Quercus Brantii Lind 1 in Zagros forests. Forest Research and Development, 1(3): 231-240 (In Persian).

25. Modaberi, A., J. Soosani, K. Abrari Vajari, Sh. Khosravi and P. Farhadi. 2016. The Survey of the Structure of the Central Zagros Forests. Forest Strategical Approchment Journal. 1(3): 34-45 (In Persian). 
26. Mouro, S.M., L.V. García, T. Marañón and H. Freitas. 2007. Recruitment Patterns in a Mediterranean Oak Forest: A Case Study Showing the Importance of the Spatial Component. Forest Science, 53(6): 645-652.

27. Palmino, R.L. 2005. Spatial distribution patterns of trees in a seasonally dry forest in the ceros de Amotape National Park, northwestern Peru. Revista Peruana de Biologia, 12(2): 317-326.

28. Porma, R., Sh. Shataee Joybari, Y. Khodakarami, H. Habashi. 2010. Evaluation of Landsat-ETM+ and IRS-LISS III satellite data for forest type mapping in Zagros forest (Case study: Ghalajeh forest, Kermanshah province). Iranian Journal of Forest and Poplar Research, 17(4): 594-606 (In Persian).

29. Pourreza, M., J.D. Shaw, H. Zangeneh. 2008. Sustainability of wild pistachio (Pistacia atlantica Desf.) in Zagros forests, Iran. Forest Ecology and Management, 225: 3667-3671.

30. Rayburn, A.P. 2011. Causes and Consequences of Plant Spatial Patterns in Natural and Experimental Great Basin (USA) Plant Communities. PhD thesis, Utah State University, 162 pp.

31. Rayburn, A.P., K. Schiffers and E.W. Schupp. 2011. Use of precise spatial data for describing spatial patterns and plant interactions in a diverse Great Basin shrub community. Plant Ecology, 212: 585594.

32. Ripley, B.D. 1977. Modeling spatial patterns. Journal of the Royal Statistical Society, Series B (Methodological), 39(2): 172-212.

33. Safari, A. 2009. The Study of Spatial Pattern of Oak manna tree (Quercus brantii Lindl.) and Wild pistachio (Pistacia atlantica Desf.) in Zagross Forests. (Case Study: Bayangan, Kermanshah province). M.Sc. Thesis, University of Kurdistan, Sanandaj, Iran. 154 pp (In Persian).

34. Schiffers, K., F.M. Schurr, K. Tielbörger, C. Urbach, K. Moloney and F. Jeltsch. 2008. Dealing with virtual aggregation- a new index for analyzing heterogeneous point patterns. Ecography, 31: 545-555.

35. Stamatellos, G. and G. Panourgias. 2005. Simulating spatial distributions of forest trees by using data from fixed area plots. Forestry, 78(3): 305-312.

36. Tsitsoni, T., D. Karamanolis, G. Stamatellos and P. Ganatsas. 2003. Spatial pattern and connection of tree diameter classes in Pinus halepensis M. Stands after wildfire. $8^{\text {th }}$ International conference on environmental science and technology Lemons Island, Greece, 904-910.

37. Watson, D.M., D.A. Rosheir and T. Wiegand. 2007. Spatial ecology of a root parasite- from pattern to process. Austral Ecology, 32: 359-369.

38. Wei-dong, H., G. Xiu-mei and L. Lin-feng. 2001. Spatial pattern of dominant species of the secondary monsoon rain forest in Lianjiang, Guangdong Province. Journal of Forestry Research, 12(2): 101-104.

39. Wiegand, K., F. Jeltsch and D. Ward. 2000. Do spatial effects play a role in the spatial distribution of desert-dwelling Acacia raddiana?, Journal of Vegetation Science, 11: 473-484.

40. Wiegand, T. and K.A. Moloney. 2004. Rings, circles and null-models for point pattern analysis in ecology. Oikos, 104: 209-229.

41. Wiegand, T. and K.A. Moloney. 2014. Handbook of Spatial Point-Pattern Analysis in Ecology. Taylor and Francis Group, CRC Press, 538 pp.

42. Wiegand, T., S. Gunatilleke, N. Gunatilleke and T. Okuda. 2007. Analysing the syructure of a Sri lankan trees Species with multiple scales of clustering. Ecology, 88(12): 3088-3102.

43. Zhang, Q., Y. Zhang, S. Peng, E. Yirdaw and N. Wu. 2009. Spatial structure of Alpine trees in mountain Baima Xueshan on the southeast Tibetan plateau. Silva Fennica, 43(2): 197-208.

44. Zhang, Y., J. Li, Sh. Chang, X. Li and J. Lu. 2012. Spatial distribution pattern of Picea schrenkiana population in the Middle Tianshan Mountains and the relationship with topographic attributes. Journal of Arid Land, 4(4): 457-468.

45. Zhu, Y., S. Getzin, T. Wiegand, H. Ren and K. Ma. 2013. The Relative Importance of Janzen-Connell Effects in Influencing the Spatial Patterns at the Gutianshan Subtropical Forest. Plos One, 8(9): e74560. doi:10.1371/journal.pone.0074560. 


\title{
Spatial Pattern and Interaction Analysis of Quercus Brantii Lindl. and Pistacia Atlantica Desf. in Qalajeh Forests of Kermanshah using K2 Function
}

\author{
Mahsa Karimi ${ }^{1}$ and Asghar Fallah ${ }^{2}$ \\ 1- Ph.D. Student, Sari Agricultural Sciences and Natural Resources University, \\ (Corresponding author: karimi.mahsa99@gmail.com) \\ 2- Associate Professor, Sari Agricultural Sciences and Natural Resources University \\ Received: June 27, 2017 Accepted: December 4, 2017
}

\begin{abstract}
Quercus brantii Lindl. and Pistacia atlantica Desf. are the most important tree species in Zagros forests, The abundant use of these trees by the inhabitants of the area has led to a reduction in the quality and quantity of these valuable species, as well as the creation of heterogeneous masses.Recognizing the spatial pattern and the interactions of trees can be a key to managerial interventions for the conservation, restoration and development of forests, On the other hand, the continued involvement of Zagros inhabitants in forest areas has created many heterogeneous areas that make it necessary to recognize these areas in order to manage them in order to reduce the impact of human intervention. Therefore, the aim of this study is to determine the spatial pattern and interactions of Quercus brantii Lindl. and Pistacia atlantica Desf. in heterogeneous masses of Zagros forests. For this purpose, an heterogeneous area of 30 hectares was selected in Kermanshah Qalajeh forests and fully was inventoried, A point map of Quercus brantii Lindl. trees and Pistacia atlantica Desf. in the study area was prepared using a distance-azimuth method. In this study, the spatial pattern and interaction of trees was analyzed using univariate and bivariate K2 function. The results of this study showed that Quercus brantii Lindl. trees and Pistacia atlantica Desf. are $85.6 \%$ and $9.7 \%$ of the trees in the studied area and have a mixed pattern. Spatial pattern of Quercus brantii Lindl. and Pistacia atlantica Desf. changes from clumped to random and from random to uniform with increasing of distance, respectively. Analysis of K2 bivariate function showed attraction in distances of less than 8 meters and the non-interaction of species in other distances.
\end{abstract}

Keywords: Point map, Heterogeneous mass, Distance-azimuth method, Monte-Carlo range, Clumped pattern 